\title{
Determination of Bioefficacy and Phytotoxicity of Fungicide Taqat 75\% WP (Captan 70\% + Hexaconazole 5\%) against Leaf Spot and Fruit Spot Diseases in Tomato Crop
}

\author{
Rishu Sharma* \\ Department of Plant Pathology, Faculty of Agriculture, Bidhan Chandra Krishi \\ Viswavidyalaya, Mohanpur, 741252, Dist: Nadia. West Bengal, India \\ *Corresponding author
}

\begin{tabular}{|c|}
\hline Keywords \\
\hline $\begin{array}{l}\text { Tomato, Leaf spot } \\
\text { Fruit spot, } \\
\text { Plant diseases }\end{array}$ \\
\hline Article Info \\
\hline $\begin{array}{l}\text { Accepted: } \\
\text { 07 March } 2020 \\
\text { Available Online: } \\
10 \text { April } 2020\end{array}$ \\
\hline
\end{tabular}

A B S T R A C T

Several pathogens attack the tomato cropduring its cropping season. The present experiments were carried out to study the disease incidence using Taqat 75\% WP during the year 2016-17 and 2017-18. Assessment of the disease severity was done by standard scoring methods and expressed as Per cent Disease Index (PDI). For phytotoxicity of Taqat $75 \% \mathrm{WP}$ at X dose $750 \mathrm{~g} / \mathrm{ha}, 2 \mathrm{X}$ dose $1500 \mathrm{~g} / \mathrm{ha}$ and $4 \mathrm{X}$ doses $3000 \mathrm{~g} / \mathrm{ha}$ along with the standard check treatments. The observations on leaf injury, wilting, vein clearing, necrosis, epinasty \& hyponasty were recorded on ten randomly selected plants before spray at 3,7 and 15 days after $1^{\text {st }}$ spray. The observations were recorded on leaf spot and fruit spot before application and at different intervals after the application of Taqat $75 \% \mathrm{WP}$. Thus, our studies revealed that the per cent disease index (PDI) of Taqat 75\%WP (Captan $70 \%+$ Hexaconazole 5\%) @ $750 \mathrm{~g} / \mathrm{ha}$ is highly effective in controlling leaf spot and fruit spot diseases of tomato and increasing tomato fruit yield without causing any phytotoxicity to the crop.

\section{Introduction}

Tomato (Solanum lycopersicum L.) is a highvalue dietary component, contributing to nutrition and livelihood of both rural and urban population the world over. Tomato is an obligatory enthusiastic vegetable and fruit crop belongs to Solanaceae family. It is the most important tropical vegetable crop widely used throughout the world (Hadian et al., 2011). It is rich in vitamins, minerals, organic acids, and also containing considerable amount of total sugar (3\% 4\%), total solids (4\% 7\%), ascorbic acid (15 30 mg/100g), and lycopene (20 50 mg/100g) (Giovannucci, 1999). It is a high-value horticultural crop for 
the local market and an important dietary component, contributing to improved nutrition and livelihood for both rural and urban population (Waiganjo et al., 2006). India is one of the major producers of tomato where 0.865 mha area is under tomato cultivation producing 16.826 million tonnes with an average productivity 19.5 tonnes per ha (Shiva et al., 2013). The fruits are used fresh in salads or cooked as a vegetable, in processed form as tomato paste (puree), tomato sauce, ketchup, juice and can also be dried. They are rich in vitamins $\mathrm{A}$ and $\mathrm{C}$ and are gaining importance because it contains lycopene, a food component known to reduce the incidence of prostate cancer, heart and age related diseases (AVRDC, 2003).

Tomato is fairly adaptable, but grows well in warm conditions with optimum temperatures of $15^{\circ} \mathrm{C}-25^{\circ} \mathrm{C}$. But, it is attacked by several plant pathogens causing viz. Leaf spot (Septoria lycopersici) and fruit spot (Phytophthora parasitica). In the present Study, disease incidence and phytotoxicity studies using Taqat $75 \%$ WP during the year 2016-17 and 2017-18 were carried. Assessment of the disease severity was done by standard scoring methods, and expressed as Per cent Disease Index (PDI).

For phytotoxicity of Taqat $75 \% \mathrm{WP}$ at $\mathrm{X}$ dose $750 \mathrm{~g} / \mathrm{ha}, 2 \mathrm{X}$ dose $1500 \mathrm{~g} / \mathrm{ha}$ and $4 \mathrm{X}$ doses $3000 \mathrm{~g} / \mathrm{ha}$ along with the standard check treatments. The observations on leaf injury, wilting, vein clearing, necrosis, epinasty \& hyponasty were recorded on ten randomly selected plants before spray, 3, 7 and 15 days after $1^{\text {st }}$ spray.

\section{Materials and Methods}

A local tomato variety Patharkuchi was grown during Rabi, 2016-17 and 2017-18 and the experimental layout was made with nine treatments and three replications in RBD design for the bioefficacy studies with a spacing of $60 \times 40 \mathrm{~cm}^{2}$ and six treatments for the phytotoxicity studies. All the agronomical practices were followed as per the standard package of practices recommendations. Assessment of the disease severity was done by scoring methods as given below and expressed as Per cent Disease Index (PDI).

\section{Bio-efficacy of taqat}

On appearance of the diseases, fungicides were sprayed with knapsack sprayer fitted with hollow cone nozzle. The per cent disease incidence on leaves and fruits were recorded before spray initiation and at different intervals after each spray on randomly selected 25 leaves of five plants. Assessment of the disease severity was done by scoring methods as given below and expressed as Per cent Disease Index (PDI) (Table 1\& 2).

\section{The percent disease index was calculated} by using the formula

$$
\text { PDI }=\frac{\text { Sum of all disease Ratings }}{\text { Total no. of leaves assessed } \times \text { Maximum Disease grade }} \times 100
$$

\section{Disease scoring scale for fruit spot}

Fruit spot was assessed based on the total number of fruits in 10 randomly selected plants and number of infected fruits in each plot. Later it was converted to per cent infected fruit and presented in Table 3.

\section{Phytotoxicity of taqat on tomato}

For phytotoxicity of Taqat $75 \% \mathrm{WP}$ at $\mathrm{X}$ dose $750 \mathrm{~g} / \mathrm{ha}, 2 \mathrm{X}$ dose $1500 \mathrm{~g} / \mathrm{ha}$ and $4 \mathrm{X}$ doses $3000 \mathrm{~g} / \mathrm{ha}$ along with the standard check treatments. All the field experimental conditions were kept constant. Observations on leaf injury, wilting, vein clearing, necrosis, epinasty \& hyponasty were recorded on ten randomly selected plants before spray, 3, 7 and 15 days after $1^{\text {st }}$ spray. The level of 
phytotoxicity was estimated by visual assessment on below mentioned scale of 0-10.

\section{Experimental results}

The observations on major diseases of tomato viz. leaf spot and fruit spot before application and at different intervals after the application of Taqat 75\%WP @750 g/ha, its individual products and the standard check fungicides, Captan 50\%WP @ 2500 g/ha and Mancozeb 75\%WP @ $1500 \mathrm{~g} / \mathrm{ha}$ along with the untreated control treatment were recorded. The per cent disease index was worked out was analyzed statistically. The data on leaf spot and fruit spot were presented in tables 1 , $2,3 \& 4$ respectively and the results are discussed below.

\section{Leaf spot disease of tomato}

At the time of spray initiation the Per cent Disease Index (PDI) was between $4.89 \& 7.56$ during two cropping seasons in the year 201617 and 2017-18. After the application of fungicides, all the fungicides exhibited significantly superior efficacy against leaf spot disease.

After two applications in the year 2016-17, the mean PDI was minimum in the treatment, Taqat 75\% WP @ 750g/ha recording (7.67 PDI) which was followed by Taqat $75 \%$ WP @ 500g/ha (10.67 PDI) and Hexaconazole $5 \%$ EC @ 750ml/ha (11.00 PDI), Hexaconazole 5\%EC @ 500ml/ha (12.93 PDI).

The standard checks treatments, Mancozeb 75\%W P @ 1500g/ha recorded 14.37 PDI and Captan 50\% WP @ 2500g/ha recorded 16.04 PDI. Maximum PDI of 48.33 was recorded in untreated control (Table 4).
After two applications in the year 2017-18, the mean PDI was minimum in the treatment, Taqat 75\%WP @ 750g/ha recording 6.85 which was followed by Taqat 75\%WP @ 500g/ha (8.56 PDI) and Hexaconazole 5\%EC @ 750ml/ha (9.00 PDI), Hexaconazole 5\%EC @ 500ml/ha (10.52 PDI). The standard checks treatments, Mancozeb 75\%WP @ $1500 \mathrm{~g} / \mathrm{ha}$ recorded 11.89 PDI and Captan 50\%WP @ 2500g/ha recorded 12.37 PDI. Maximum PDI of 48.85 was recorded in untreated control (Table 5).

\section{Fruit spot disease of tomato}

Fruit spot disease observation was recorded at 15 days after $2^{\text {nd }}$ spray during the year 201617. Among all the treatments, the minimum of $4.70 \%$ fruit spots was in Taqat 75\%WP @ $750 \mathrm{~g} / \mathrm{ha}$. which was followed by Taqat 75\%WP@ 500g/ha (6.69\%), Hexaconazole $5 \%$ EC @ 750ml/ha (6.83\%) and Hexaconazole 5\%EC @ 500ml/ha (8.29\%). The standard checks treatments, Mancozeb 75\%WP @ 1500g/ha recorded 10.40\% and Captan 50\%WP @ 2500g/ha recorded 9.94\%. Maximum of $27.70 \%$ was recorded in untreated control (Table 3).

Fruit spot disease observation was recorded at 15 days after $2^{\text {nd }}$ spray during the year 201718. Among all the treatments, the minimum of $5.10 \%$ fruit spots was in Taqat 75\%WP @ $750 \mathrm{~g} / \mathrm{ha}$. Which was followed by Taqat $75 \%$ WP @ 500g/ha (7.13\%), Hexaconazole 5\% EC@ $@ 750 \mathrm{ml} / \mathrm{ha}(7.99 \%)$ and Hexaconazole 5\% EC@500ml/ha (8.87\%). The standard checks treatments, Mancozeb 75\%WP @ $1500 \mathrm{~g} / \mathrm{ha}$ recorded $10.28 \%$ and Captan $50 \%$ WP @ 2500g/ha recorded 10.34\%. Maximum of $29.46 \%$ was recorded in untreated control (Table 3). 
Table.1 Disease scoring scale for leaf spot

\begin{tabular}{|c|c|}
\hline Score & Symptoms \\
\hline 0 & No symptoms on leaf \\
\hline 1 & Very small, irregular spots covering less than $1 \%$ of leaf area \\
\hline 3 & Small irregular, brown spots covering $1-10 \%$ of the leaf area \\
\hline 5 & $\begin{array}{l}\text { Small lesions, but enlarged with dark brown margin with a grey center covering } \\
11-25 \% \text { of the leaf area }\end{array}$ \\
\hline 7 & $\begin{array}{l}\text { Enlarged lesions, with dark brown margin with a grey center covering } 26-50 \% \text { of } \\
\text { the leaf area. }\end{array}$ \\
\hline 9 & $\begin{array}{l}\text { Lesions coalesce to from irregular dark brown to black patches covering } 51 \% \text { or } \\
\text { more of the leaf area. }\end{array}$ \\
\hline
\end{tabular}

Table.2 Scale for phytotoxicity

\begin{tabular}{|c|c|}
\hline Score & Phytotoxicity (\%) \\
\hline $\mathbf{0}$ & No phytotoxicity \\
\hline $\mathbf{1}$ & $1-10$ \\
\hline $\mathbf{2}$ & $11-20$ \\
\hline $\mathbf{3}$ & $21-30$ \\
\hline $\mathbf{4}$ & $31-40$ \\
\hline $\mathbf{5}$ & $41-50$ \\
\hline $\mathbf{6}$ & $51-60$ \\
\hline $\mathbf{7}$ & $61-70$ \\
\hline $\mathbf{8}$ & $71-80$ \\
\hline $\mathbf{9}$ & $81-90$ \\
\hline $\mathbf{1 0}$ & $91-100$ \\
\hline
\end{tabular}

Table.3 Effect of Taqat 75\% WP against Fruit spot (Alternaria sp.) in Tomato during Rabi, 2016-17 and 2017-18

\begin{tabular}{|c|c|c|}
\hline Tr. No & Mean $1^{\text {st }}$ year & $\begin{array}{c}\text { Mean } 2^{\text {nc }} \\
\text { year }\end{array}$ \\
\hline T1 & 10.67 & 8.56 \\
\hline T2 & 7.67 & 6.85 \\
\hline T3 & 21.63 & 15.48 \\
\hline T4 & 18.89 & 13.78 \\
\hline T5 & 16.04 & 12.37 \\
\hline T6 & 12.93 & 10.52 \\
\hline T7 & 11.00 & 9.00 \\
\hline T8 & 14.37 & 11.89 \\
\hline T9 & 48.33 & 48.85 \\
\hline
\end{tabular}


Fig.1 Effect of Taqat 75\% WP against Fruit spot (Alternaria sp.) in Tomato during Rabi, 2016-17 and 2017-18

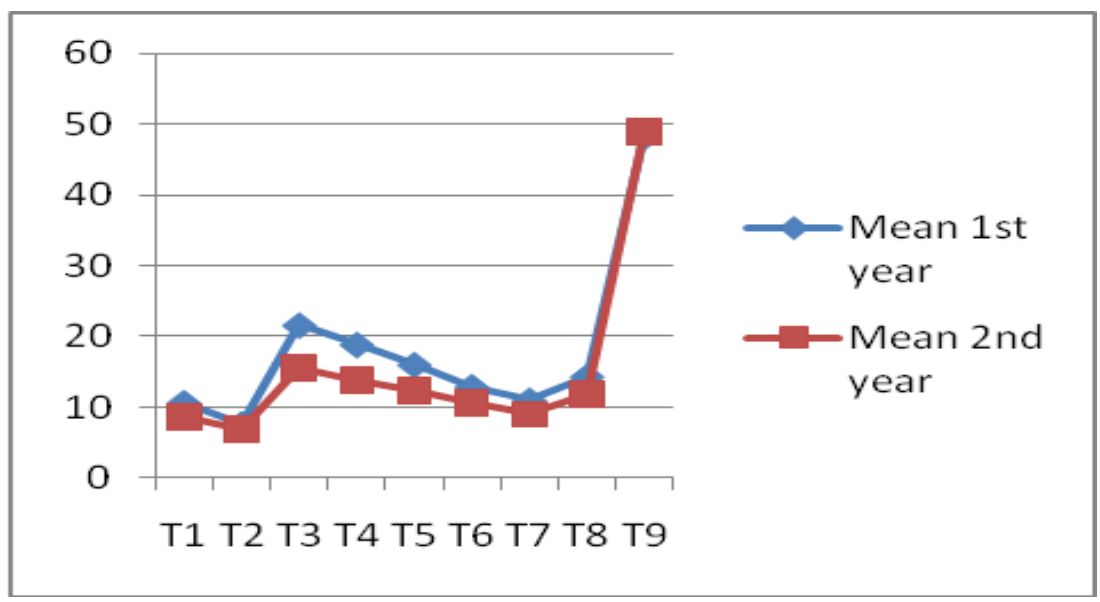

Table.4 Effect of Taqat 75\% WP against Leaf spot in Tomato during Rabi, 2016-17

\begin{tabular}{|c|c|c|c|c|c|c|c|c|c|}
\hline \multirow{2}{*}{$\begin{array}{l}\text { Tr. } \\
\text { No }\end{array}$} & \multirow[t]{2}{*}{ Treatments } & \multirow{2}{*}{$\begin{array}{c}\text { Dose/ha } \\
\text { (Formul } \\
\text { ation) }\end{array}$} & \multicolumn{5}{|c|}{ Per cent Disease Index (PDI) } & \multirow[t]{2}{*}{ Mean } & \multirow{2}{*}{$\begin{array}{c}\% \\
\text { control }\end{array}$} \\
\hline & & & $\begin{array}{c}\text { Pre- } \\
\text { treatment }\end{array}$ & $\begin{array}{c}10 \text { Days } \\
\text { After 1st } \\
\text { spray }\end{array}$ & $\begin{array}{c}15 \text { Days } \\
\text { After 1st } \\
\text { spray }\end{array}$ & $\begin{array}{l}10 \text { Days } \\
\text { After 2nd } \\
\text { spray }\end{array}$ & $\begin{array}{c}15 \text { Days } \\
\text { After } \\
\text { 2nd } \\
\text { spray }\end{array}$ & & \\
\hline $\mathbf{T 1}$ & $\begin{array}{l}\text { Taqat } \\
75 \% \mathrm{WP}\end{array}$ & $500 \mathrm{~g}$ & $\begin{array}{c}6.22 \\
(14.42)\end{array}$ & $\begin{array}{c}7.26 \\
(15.60)\end{array}$ & $\begin{array}{c}10.67 \\
(18.93)\end{array}$ & $\begin{array}{c}11.56 \\
(19.84)\end{array}$ & $\begin{array}{c}13.19 \\
(21.25)\end{array}$ & 10.67 & 77.93 \\
\hline $\mathbf{T 2}$ & $\begin{array}{l}\text { Taqat } \\
75 \% \mathrm{WP}\end{array}$ & $750 \mathrm{~g}$ & $\begin{array}{c}5.48 \\
(13.53)\end{array}$ & $\begin{array}{c}6.07 \\
(14.24)\end{array}$ & $\begin{array}{c}7.26 \\
(15.44)\end{array}$ & $\begin{array}{c}8.15 \\
(16.56)\end{array}$ & $\begin{array}{c}9.19 \\
(17.55)\end{array}$ & 7.67 & 84.14 \\
\hline T3 & $\begin{array}{l}\text { Captan } \\
50 \% \mathrm{WP}\end{array}$ & $700 \mathrm{~g}$ & $\begin{array}{c}5.78 \\
(13.90)\end{array}$ & $\begin{array}{c}16.89 \\
(24.18)\end{array}$ & $\begin{array}{c}21.19 \\
(27.39)\end{array}$ & $\begin{array}{c}23.11 \\
(28.70)\end{array}$ & $\begin{array}{c}25.33 \\
(30.21)\end{array}$ & 21.63 & 55.25 \\
\hline $\mathbf{T 4}$ & $\begin{array}{l}\text { Captan } \\
50 \% \mathrm{WP}\end{array}$ & $1050 \mathrm{~g}$ & $\begin{array}{c}6.07 \\
(14.25)\end{array}$ & $\begin{array}{c}13.93 \\
(21.88)\end{array}$ & $\begin{array}{c}18.52 \\
(25.47)\end{array}$ & $\begin{array}{c}20.44 \\
(26.87)\end{array}$ & $\begin{array}{c}22.67 \\
(28.40)\end{array}$ & 18.89 & 60.92 \\
\hline T5 & $\begin{array}{l}\text { Captan } \\
50 \% \mathrm{WP}\end{array}$ & $2500 \mathrm{~g}$ & $\begin{array}{c}6.22 \\
(14.42)\end{array}$ & $\begin{array}{c}11.41 \\
(19.67)\end{array}$ & $\begin{array}{c}15.56 \\
(23.21)\end{array}$ & $\begin{array}{c}17.63 \\
(24.80)\end{array}$ & $\begin{array}{c}19.56 \\
(26.23)\end{array}$ & 16.04 & 66.82 \\
\hline T6 & $\begin{array}{l}\text { Hexaconazol } \\
\text { e } 5 \% \text { EC }\end{array}$ & $500 \mathrm{ml}$ & $\begin{array}{c}7.11 \\
(15.45)\end{array}$ & $\begin{array}{c}9.19 \\
(17.59)\end{array}$ & $\begin{array}{c}12.00 \\
(20.19)\end{array}$ & $\begin{array}{c}14.67 \\
(22.50)\end{array}$ & $\begin{array}{c}15.85 \\
(23.43)\end{array}$ & 12.93 & 73.26 \\
\hline T7 & $\begin{array}{l}\text { Hexaconazol } \\
\text { e } 5 \% \text { EC }\end{array}$ & $750 \mathrm{ml}$ & $\begin{array}{c}5.04 \\
(12.35)\end{array}$ & $\begin{array}{c}7.70 \\
(16.10)\end{array}$ & $\begin{array}{c}10.67 \\
(19.05)\end{array}$ & $\begin{array}{c}12.00 \\
(20.22)\end{array}$ & $\begin{array}{c}13.63 \\
(21.65)\end{array}$ & 11.00 & 77.24 \\
\hline T8 & $\begin{array}{l}\text { Mancozeb } \\
75 \% \text { WP }\end{array}$ & $1500 \mathrm{~g}$ & $\begin{array}{c}4.89 \\
(11.94)\end{array}$ & $\begin{array}{c}10.81 \\
(19.19)\end{array}$ & $\begin{array}{c}13.63 \\
(21.64)\end{array}$ & $\begin{array}{c}15.85 \\
(23.45)\end{array}$ & $\begin{array}{c}17.19 \\
(24.47)\end{array}$ & 14.37 & 70.27 \\
\hline T9 & $\begin{array}{l}\text { Untreated } \\
\text { control }\end{array}$ & - & $\begin{array}{c}7.56 \\
(15.95)\end{array}$ & $\begin{array}{c}31.11 \\
(33.85)\end{array}$ & $\begin{array}{c}49.04 \\
(44.43)\end{array}$ & $\begin{array}{c}55.26 \\
(48.01)\end{array}$ & $\begin{array}{c}57.93 \\
(49.55)\end{array}$ & 48.33 & \\
\hline & S.Em \pm & & 1.49 & 0.96 & 1.16 & 0.84 & 0.88 & & \\
\hline & CD at $5 \%$ & & 4.46 & 2.88 & 3.48 & 2.52 & 2.63 & & \\
\hline
\end{tabular}

Figures in the parenthesis are angular transformed values 
Table.5 Effect of Taqat 75\% WP against Leaf spot in Tomato during Rabi, 2017-18

\begin{tabular}{|c|c|c|c|c|c|c|c|c|c|}
\hline \multirow{2}{*}{$\begin{array}{l}\text { Tr. } \\
\text { No }\end{array}$} & \multirow[t]{2}{*}{ Treatments } & \multirow{2}{*}{$\begin{array}{l}\text { Dose/ha } \\
\text { (Formula } \\
\text { tion) }\end{array}$} & \multicolumn{5}{|c|}{ Per cent Disease Index (PDI) } & \multirow[t]{2}{*}{ Mean } & \multirow[t]{2}{*}{$\%$ control } \\
\hline & & & $\begin{array}{c}\text { Pre- } \\
\text { treatmen } \\
\mathrm{t}\end{array}$ & $\begin{array}{c}10 \text { Days } \\
\text { After 1st } \\
\text { spray }\end{array}$ & $\begin{array}{l}15 \text { Days } \\
\text { After 1st } \\
\text { spray }\end{array}$ & $\begin{array}{l}10 \text { Days } \\
\text { After } 2 \text { nd } \\
\text { spray }\end{array}$ & $\begin{array}{l}15 \text { Days } \\
\text { After 2nd } \\
\text { spray }\end{array}$ & & \\
\hline T1 & $\begin{array}{l}\text { Taqat } \\
75 \% \mathrm{WP}\end{array}$ & $500 \mathrm{~g}$ & $\begin{array}{c}4.89 \\
(12.69)\end{array}$ & $\begin{array}{c}6.52 \\
(14.63)\end{array}$ & $\begin{array}{c}7.41 \\
(15.70)\end{array}$ & $\begin{array}{c}9.63 \\
(18.06)\end{array}$ & $\begin{array}{c}10.67 \\
(19.02)\end{array}$ & 8.56 & 82.49 \\
\hline $\mathbf{T} 2$ & $\begin{array}{l}\text { Taqat } \\
75 \% \mathrm{WP}\end{array}$ & $750 \mathrm{~g}$ & $\begin{array}{c}5.19 \\
(13.15)\end{array}$ & $\begin{array}{c}5.78 \\
(13.88)\end{array}$ & $\begin{array}{c}6.07 \\
(14.22)\end{array}$ & $\begin{array}{c}7.41 \\
(15.66)\end{array}$ & $\begin{array}{c}8.15 \\
(16.48)\end{array}$ & 6.85 & 85.97 \\
\hline T3 & $\begin{array}{l}\text { Captan } \\
50 \% \mathrm{WP}\end{array}$ & $700 \mathrm{~g}$ & $\begin{array}{c}5.04 \\
(12.95)\end{array}$ & $\begin{array}{c}12.30 \\
(20.22)\end{array}$ & $\begin{array}{c}14.67 \\
(22.42)\end{array}$ & $\begin{array}{c}16.74 \\
(24.13)\end{array}$ & $\begin{array}{c}18.22 \\
(25.13)\end{array}$ & 15.48 & 68.31 \\
\hline T4 & $\begin{array}{l}\text { Captan } \\
50 \% \mathrm{WP}\end{array}$ & $1050 \mathrm{~g}$ & $\begin{array}{c}4.89 \\
(12.76)\end{array}$ & $\begin{array}{c}10.67 \\
(18.98)\end{array}$ & $\begin{array}{c}12.89 \\
(21.03)\end{array}$ & $\begin{array}{c}14.67 \\
(22.49)\end{array}$ & $\begin{array}{c}16.89 \\
(24.24)\end{array}$ & 13.78 & 71.80 \\
\hline T5 & $\begin{array}{l}\text { Captan } \\
50 \% \mathrm{WP}\end{array}$ & $2500 \mathrm{~g}$ & $\begin{array}{c}5.19 \\
(13.15)\end{array}$ & $\begin{array}{c}9.78 \\
(17.94)\end{array}$ & $\begin{array}{c}11.56 \\
(19.80)\end{array}$ & $\begin{array}{c}13.33 \\
(21.37)\end{array}$ & $\begin{array}{c}14.81 \\
(22.50)\end{array}$ & 12.37 & 74.68 \\
\hline T6 & $\begin{array}{l}\text { Hexaconazo } \\
\text { le } 5 \% \text { EC }\end{array}$ & $500 \mathrm{ml}$ & $\begin{array}{c}5.19 \\
(13.15)\end{array}$ & $\begin{array}{c}7.85 \\
(16.24)\end{array}$ & $\begin{array}{c}9.93 \\
(18.35)\end{array}$ & $\begin{array}{c}11.56 \\
(19.84)\end{array}$ & $\begin{array}{c}12.74 \\
(20.88)\end{array}$ & 10.52 & 78.47 \\
\hline T7 & $\begin{array}{l}\text { Hexaconazo } \\
\text { le } 5 \% \mathrm{EC}\end{array}$ & $750 \mathrm{ml}$ & $\begin{array}{c}5.48 \\
(13.52)\end{array}$ & $\begin{array}{c}6.96 \\
(15.27)\end{array}$ & $\begin{array}{c}8.59 \\
(16.81)\end{array}$ & $\begin{array}{c}9.78 \\
(18.11)\end{array}$ & $\begin{array}{c}10.67 \\
(19.05)\end{array}$ & 9.00 & 81.58 \\
\hline T8 & $\begin{array}{l}\text { Mancozeb } \\
75 \% \text { WP }\end{array}$ & $1500 \mathrm{~g}$ & $\begin{array}{c}5.19 \\
(13.15)\end{array}$ & $\begin{array}{c}8.89 \\
(17.33)\end{array}$ & $\begin{array}{c}11.41 \\
(19.72)\end{array}$ & $\begin{array}{c}13.04 \\
(21.16)\end{array}$ & $\begin{array}{c}14.22 \\
(22.14)\end{array}$ & 11.89 & 75.66 \\
\hline T9 & $\begin{array}{l}\text { Untreated } \\
\text { control }\end{array}$ & - & $\begin{array}{c}6.81 \\
(14.90)\end{array}$ & $\begin{array}{c}35.85 \\
(36.75)\end{array}$ & $\begin{array}{c}46.67 \\
(43.06)\end{array}$ & $\begin{array}{c}53.78 \\
(47.15)\end{array}$ & $\begin{array}{c}59.11 \\
(50.23)\end{array}$ & 48.85 & \\
\hline & S.Em \pm & & 0.82 & 1.57 & 1.24 & 0.97 & 1.24 & & \\
\hline & CD at $5 \%$ & & 2.47 & 4.70 & 3.71 & 2.91 & 3.73 & & \\
\hline
\end{tabular}

Figures in the parenthesis are angular transformed values

\section{Effect on yield}

All the fungicidal treatments showed the impact on the increase in the fruit yield. Maximum fruit yield of 28.76 tones/ha was recorded in Taqat 75\%WP @ 750g/ha. Which was followed by its lower dose of $500 \mathrm{~g} / \mathrm{ha}$ (27.73 tones/ha), Hexaconazole 5\%EC @ $750 \mathrm{ml} / \mathrm{ha}$ recorded 27.26 tones/ha, Captan 50\%WP @ 2500g/ha recorded 26.79 tones/ha, Captan 50\%WP @ 1050g/ha recorded 26.61 tones/ha and Minimum fruit yield of 22.56 tones was recorded in untreated control (Fig. 1).

Based on experimental results, it can be concluded that Taqat 75\%WP (Captan 70\% + Hexaconazole 5\%) @ $750 \mathrm{~g} / \mathrm{ha}$ is highly effective in controlling leaf spot and fruit spot diseases of tomato and increasing tomato fruit yield. Application of Taqat $75 \% \mathrm{WP}$ is not causing any phytotoxicity to the crop.

\section{Acknowledgement}

I highly grateful to funding agency Rallis India Pvt. Ltd, Bangalore, India and BCKV for conducting the cropping trials in the university farm.

\section{References}

AVRDC (2003). Asian Vegetable Research and Development Corporation, Progress Report. Variations of anti-oxidants and their activity in tomato. Pp. 70-115.

Giovannucci, E. 1999. Tomatoes, tomato 
based products, lycopene and cancer: Review of the epidemiologic literature. J. Nat. Cancer Inst. 91:317-331

Hadian, S., Rahnam, K., Jamali, S. and Escandari, A. 2011. Comparing New Extract with Chemical Control on Fusarium oxysporum I and Meloidogyne incognita complex of tomato. Advances in Environmental Biology. 5(8): 2052-2057

Shiva. N., G. Gomathi, S. Karthika, S. Ramya, B. Senathipathi, P. Senthil, K.
K. Surenda and S.R. Kumar. 2013. Physiological Effects of Pseudomonas fluorescens on tomato. Int. J. Hort.3(18):104-108.

Waiganjo, M.M., Wabule,N.M., Nyongesa, D., Kibaki, J.M., Onyango, I., Webukhulu, S.B. and Muthoka, N.M. (2006). Tomato production in Kiriyanga District, Kenya. A baseline survey report. KARI/IPM-CRSP Collaborative project.

\section{How to cite this article:}

Rishu Sharma. 2020. Determination of Bioefficacy and Phytotoxicity of Fungicide Taqat 75\% WP (Captan 70\% + Hexaconazole 5\%) against Leaf Spot and Fruit Spot Diseases in Tomato Crop. Int.J.Curr.Microbiol.App.Sci. 9(04): xx-xx. doi: https://doi.org/10.20546/ijcmas.2020.904.051 